\title{
Wege des völkerrechtlichen Umgangs mit Kampfdrohnen
}

\author{
Rolf Mützenich und David Bieger*
}

\begin{abstract}
Due to both the growing number of military operations carried out by unmanned combat air vehicles (UCAVs) and the increase in production of UCAVs, a response of the international community is imperative. But what is the adequate reaction on this futuristic drone technology? After pointing out the danger of the trend to full autonomy of UCAVs and existing initiatives against such a development, this paper turns the spotlight to Public International Law and the question whether a general prohibition of UCAV missions already exists. Furthermore, it describes effective steps for the inclusion of UCAVs in existing treaty regimes like the Treaty on Conventional Armed Forces in Europe, the Missile Technology Control Regime and the Arms Trade Treaty. The article concludes that disarmament measures and arms control instruments already exist. They only have to be used by the international community to guarantee operative global control of UCAVs.
\end{abstract}

Keywords: Combat Drone, UCAV, Public International Law, Disarmament, Arms Control Kampfdrohne, UCAV, Humanitäres Völkerrecht, Abrüstung, Rüstungskontrolle

$\mathrm{E}$ uro-Hawk-Debatte und CIA-Drohnen im Nahen Osten, Post-Drohnen über dem Rhein und ein „Angriff“ auf die Bundeskanzlerin während einer Wahlkampfveranstaltung in Dresden:

Seit einiger Zeit schwirren die kleinen und größeren unbemannten Flugkörper durch die Medien und entfachen auf ganz unterschiedlichen Feldern Diskussionen.

Dieser Artikel widmet sich den Möglichkeiten und bereits bestehenden Instrumenten einer internationalen Abrüstungs- und Rüstungskontrollpolitik bewaffneter Drohnen.

Neben einer Auseinandersetzung mit der Frage, inwieweit das Humanitäre Völkerrecht bereits jetzt einen Einsatz von Kampfdrohnen verbietet, werden internationale Abkommen, wie etwa der KSE-Vertrag (Vertrag über Konventionelle Streitkräfte in Europa), unter die Lupe genommen und konkrete Vorhaben zur völkerrechtlichen Ächtung vollautonomer Drohnen beleuchtet.

\section{Was sind Drohnen? - Definition, Abgrenzungen und Verbreitung}

Bei einer Drohne handelt es sich um ein unbemanntes Fluggerät, das durch Fernsteuerung gelenkt oder dessen Flugbahn vorprogrammiert wird (UAV = unmanned aerial vehicle). Neben vielfachen zivilen Verwendungsmöglichkeiten, die zu nicht unerheblichen Abgrenzungsschwierigkeiten führen, ist die Drohnentechnik vor allem für militärische Einsatzzwecke interessant. So setzte in den letzten Jahren ein regelrechter Boom an militärischen Drohnenprogrammen ein, die mittlerweile von ca. 76 bis 87 Staaten betrieben werden. ${ }^{1}$

* Dr. Rolf Mützenich ist stellvertretender Vorsitzender der SPD-Bundestagsfraktion. David Bieger hat Rechtswissenschaften in Bonn und Buenos Aires studiert und absolviert derzeit seinen völkerrechtlichen Schwerpunkt an der Universität zu Köln.

Dieser Beitrag hat ein anonymes Begutachtungsverfahren durchlaufen.

1 Vgl. US Government Accountability Office (GAO), Nonproliferation-Agencies could improve information sharing and End-Use Monitoring on Unmanned Aerial Vehicle Exports, in: GAO Report to the Ranking Member, Subcommittee on National Security, Homeland Defense, and Foreign Operations, Committee on Oversight and Government Reform, House of Representatives, July 2012, S. 9: http://www. gao.gov/assets/600/593131.pdf (05.02.14); siehe auch: Taylor, Guy, U.S. intelligence warily watches for threats to U.S. now that 87 nations possess drones, in: The Washington Times, 10.11.13: http://www.washingtontimes.com/news/2013/ nov/10/skys-the-limit-for-wide-wild-world-of-drones/?page=all (05.02.14).
Bei der überwältigenden Mehrzahl dieser Drohnen handelt es sich um Aufklärungs- und Überwachungsdrohnen, die lediglich eine unterstützende Funktion für die eigentlichen Kampfeinheiten übernehmen.

Bewaffnete Drohnen (UCAV = unmanned combat aerial vehicle) werden bisher lediglich von den USA, Großbritannien und Israel in militärischen Operationen eingesetzt. ${ }^{2}$ Darüber hinaus sollen China und der Iran in der Entwicklung eigener UCAVs weit fortgeschritten sein. ${ }^{3}$

Die USA können bis dato den stärksten Fortschritt in der Entwicklung von UCAVs verzeichnen. Ihre beiden populärsten Modelle „MQ-9 Reaper" und „MQ-1B Predator" wurden bereits an Großbritannien (nur Reaper) und Italien exportiert. ${ }^{4}$ Israel verfügt mit der „Heron 1" über eine eigene Aufklärungsdrohne, die mit Waffen bestückt werden kann und auch bereits eingesetzt wurde. ${ }^{5}$

In Bezug auf die Steuerung wird zwischen drei Typen differenziert, zwischen denen der Übergang jedoch oftmals fließend ist. Zur ersten Kategorie gehören UAVs, die vollständig vom Menschen kontrolliert und gesteuert werden (human-in-theloop). Bei der zweiten Kategorie befindet sich das UAV zwar unter ständiger Überwachung von Menschen, doch sind bereits weite Teile der Steuerung automatisiert. Die Entscheidung über den finalen Schuss wird aber immer erst durch menschliche Entscheidung ausgelöst und kann auch abgebrochen werden (human-on-the-loop). In der dritten Kategorie handeln die UAVs dagegen vollständig autonom, sodass selbständige Bewegungs-

2 Die USA flogen bisher Einsätze in Afghanistan, im Irak, in Somalia, Libyen, im Jemen und in Pakistan, Großbritannien setzte sie in Afghanistan ein und Israel auf der Sinai-Halbinsel (Ägypten) und im Gaza-Streifen; vgl. Taylor, 10.11.13.

3 Vgl. Franke, Ulrike Esther, Verbreitung von unbemannten Flugzeugen für den militärischen Gebrauch, in: Aus Politik und Zeitgeschichte (APuZ) 37/2013, S. 35 siehe auch: Skinner, Tony, Airshow China 2012: New UAVs designs unveiled, in: UV Online, 13.11.13: http://www.shephardmedia.com/news/uv-online/ airshow-china-2012 new-uav-designs-unveilled/ (05.02.14); Rötzer, Florian, Iran kündigt Produktionsbeginn von Kampfdrohnen an, in: Telepolis, 28.09.13 http://www.heise.de/tp/artikel/39/39998/1.html (05.02.14); Ackeret, Markus, Tarnkappen"-Drohne macht Testflug, in: Neue Zürcher Zeitung 23.11.13. http://www.nzz.ch/aktuell/international/auslandnachrichten/tarnkappendrohne-macht-testflug-1.18190484 (05.02.14).

4 Vgl. Alwardt, Christian et al., Braucht Deutschland Kampfdrohnen?, in: Hamburger Informationen zur Friedensforschung und Sicherheitspolitik, 50/2013: http://ifsh.de/tl_files/IFSH/pdf/Publikationen/ HI\%2050\%20 Kampfdrohnen. pdf (05.02.14); siehe auch: GAO, S. 11

5 Vgl. Taylor, 10.11.13. 
abläufe und Zielauswahl auch ohne menschliche Autorisierung oder Überwachung möglich sind (human-out-of-the-loop). Bisher existieren keine Human-out-of-the-loop-UCAVs, die eigenständig über den Angriff entscheiden.

\section{Proliferationseinschränkung, Abrüstung oder doch ein Verbot? - Wege des völkerrechtlichen Umgangs mit UCAVs}

Die pauschale Forderung nach einem Verbot von Drohnen muss in jedem Falle differenziert betrachtet werden. Zunächst muss konkret bestimmt werden, was genau verboten oder beschränkt werden soll: Den militärischen Einsatz von unbewaffneten UAVs generell einzuschränken oder gar zu verbieten, erscheint weder sinnvoll noch praktisch durchführbar und wird von den Diskussionsteilnehmern auch nicht anvisiert.

Hingegen findet eine Beschränkung bzw. ein vollständiges Verbot vollautonomer UCAVs (off-the-loop) unter zivilgesellschaftlichen Gruppen und in der Politik wohl den größten Anklang. ${ }^{6}$

Anders sieht es dagegen bei der Restriktion bzw. beim Verbot von nur teilautonomen oder gesteuerten UCAVs (in-the loop und on-the-loop) aus: So wird in erster Linie eine Implementierung in bestehende oder Initiativen für neue Abrüstungsabkommen und Proliferationsbeschränkungen gefordert, während einige Akteure aber auch ein generelles Verbot favorisieren.

\section{1 "The Future is now" - Bemühungen um ein Verbot vollautonomer UCAVs}

Zunächst ist zu beachten, dass bisher noch keine vollautonomen UCAVs existieren. Laut der Nichtregierungsorganisation Action on Armed Violence laufen zwar derzeit in sechs Staaten konkrete militärische Entwicklungsprogramme zu vollautonomen $\mathrm{UCAVs}^{7}$, doch sind diese bisher jedenfalls nicht über die Testphase hinaus gekommen. In den USA, die wohl den weitesten Fortschritt auf diesem Gebiet zu verzeichnen haben, besteht zudem ein zehnjähriges Moratorium für den Einsatz vollautonomer UCAVs. Eine Richtlinie des Verteidigungsministeriums erlaubt die Entwicklung vollständig autonomer Systeme nur, soweit diese nicht zu tödlicher Gewalt imstande sind. ${ }^{8}$

Dennoch befürchten viele Stimmen einen Trend hin zu vollautonomen UCAVs. ${ }^{9}$ Dabei bestehen große ethische Bedenken,

6 Vgl. etwa Deutschlands Zukunft gestalten, Koalitionsvertrag zwischen CDU, CSU und SPD, 18. Legislaturperiode, 27.11.13, S. 178: http://www.bundesregierung. de/Content/DE/_Anlagen/2013/2013-12-17-koalitionsvertrag.pdf;jsessionid $=957 \mathrm{D} 47 \mathrm{CB} 708 \mathrm{~A} 67 \mathrm{CE} 27 \mathrm{~A} 82 \mathrm{FE} 92 \mathrm{~F} 605 \mathrm{~B} 21 . \mathrm{s} 3 \mathrm{t} 2$ ?_blob=publicationFile\&v=2 (16.02.14).

7 USA, Großbritannien, China, Israel, Russland und Südkorea; Vgl. Action on Armed Violence (Campaign to Stop Killer Robots), Infographic: http://www. stopkillerrobots.org/2014/01/infographicaoav/ (06.02.14).

8 U.S. Department of Defense, Autonomy in Weapon Systems, Directive Number 3000.09, 21.11.12: http://www.dtic.mil/whs/directives/corres/pdf/300009p. pdf (06.02.14). Human Rights Watch, USA: Verbot vollständig autonomer Waffen, 16.04.13: http://www.hrw.org/de/news/2013/04/16/usa-verbotvollstaendig-autonomer-waffen (06.02.14).

9 Vgl. etwa Schörnig, Niklas und Weidlich, Christian, Keine Macht den Drohnen! in: Hessische Stiftung Friedens- und Konfliktforschung (HFSK) Standpunkte 8/2013, S. 2f: http://hsfk.de/fileadmin/downloads/standpunkt0813.pdf (05.02.14). eine Dehumanisierung der Kriegführung voranzutreiben. ${ }^{10}$ Auch könnten durch den beidseitigen Einsatz von UCAVs schnell Gewaltspiralen entstehen, die eine große Eigendynamik entfalten und der menschlichen Kontrolle entgleiten. Die Computertechnik sei zudem noch Jahrzehnte davon entfernt, ausreichende Unterscheidungen zwischen Freund und Feind zu treffen. Entsprechend wird eine weltweite Ächtung im Stile des Chemiewaffenübereinkommens oder eingebettet in die UN-Waffenkonvention forciert.

Mit der UN-Waffenkonvention (CCW = Convention on Certain Conventional Weapons) trat 1983 ein internationales Abkommen in Kraft, das die Ächtung bestimmter konventioneller Waffen zum Ziel hatte, die übermäßiges Leid verursachen oder unterschiedslos wirken.

Mit 117 Mitgliedstaaten, darunter die USA, Russland, China, Indien und Brasilien, gehört die CCW zu den wichtigsten internationalen Abrüstungsabkommen. Die CCW ist unter der Schirmherrschaft der VN als Rahmenvertrag gestaltet, dem bisher fünf Protokolle angegliedert sind. Diese untersagen beispielsweise den Einsatz blindmachender Laserwaffen oder Minen und Sprengfallen in bewaffneten Konflikten. Die fünf Protokolle sind dabei nicht abschließend und können erweitert werden, wie dies bereits bei Protokoll IV und V, die erst nachträglich hinzugefügt wurden, der Fall ist.

Die letzte Initiative für ein Protokoll VI zur Ächtung von Streumunition scheiterte 2011 an Differenzen der Mitgliedstaaten und mündete so in ein separates Abkommen (Convention on Cluster Munitions) im Jahr 2010, allerdings ohne Beteiligung wichtiger Staaten, wie die USA, Russland, China oder Brasilien.

Im November 2013 einigten sich die Mitgliedstaaten auf die Durchführung einer internationalen Konferenz im Mai 2014, um die Möglichkeiten zum Verbot vollautonomer UCAVs auszuloten. ${ }^{11}$ Diesen Schritt begrüßen Nichtregierungsorganisationen wie Human Rights Watch, die sich mit der Kampagne „Stop Killer Robots“ an die Spitze der Gegner militärischer Drohnentechnik setzten, ausdrücklich. ${ }^{12}$

\subsection{Bewertung von UCAVs im Lichte des Humanitären Völkerrechts}

Fraglich ist zunächst inwieweit UCAVs (in-the-loop und on-theloop) bereits von bestehenden Regelungen des Humanitären Völkerrechts erfasst werden.

Das Humanitäre Völkerrecht (HVR) soll durch das Verbot bestimmter Mittel und Methoden der Kriegsparteien einen weitestgehenden Schutz derjenigen Personen garantieren, die nicht oder nicht mehr am Kriegsgeschehen teilnehmen (sog. ius in bello, das Recht im Krieg, in Abgrenzung zum ius ad bellum, dem Recht zum Krieg).

Dabei bezieht es sich auf internationale und eingeschränkt auch auf nicht-internationale bewaffnete Konflikte. Es wird in erster

10 Vgl. Richter, Wolfgang, Rüstungskontrolle für Kampfdrohnen, SWP Aktuell 2013/A (29.05.13), S. 6: http://www.swp-berlin.org/de/publikationen/swpaktuell-de/swp-aktuell-detail/article/ruestungskontrolle_fuer_kampfdrohnen.html (11.02.14).

11 Human Rights Watch, UN: Nations Agree to Address ,Killer Robots', 15.11.13: http:// www.hrw.org/news/2013/11/15/un-nations-agree-address-killer-robots (10.02.14) 12 Vgl. Schörnig und Weidlich, 2013, S. 1f. 
Linie durch die Genfer Konventionen, deren Zusatzprotokolle und die Haager Landkriegsordnung bestimmt.

In den genannten Abkommen wird der Fokus jedoch nicht auf einzelne Waffenarten gelegt, sondern vielmehr auf die Art des Einsatzes und die daraus resultierenden Folgen. ${ }^{13}$ Ein generelles Verbot von UCAVs könnte sich also nur aus dem Umstand ergeben, dass diese grundsätzlich bei jedem Einsatz gegen das HVR verstoßen würden.

Gemäß Art. 22 der Haager Landkriegsordnung (HLKO) und Art. 35 Abs. 1 des Zusatzprotokolls 1 (ZP I) der HLKO sind Kriegsparteien nicht frei in der Wahl ihrer Methoden und Mittel der Kriegführung. Es gilt das Prinzip der Verhältnismäßigkeit, das eine Abwägung zwischen militärischer Notwendigkeit und zu erwartenden Folgen für die Zivilbevölkerung oder zivile Objekte verlangt und im Ergebnis zum Verbot bestimmter Waffengattungen führen kann. So sollen unnötiges Leiden und überflüssige Verletzungen verhindert und ausgeschlossen werden, wie in Art. 23 Abs. 1 lit. e HLKO und Art. 35 Abs. 2 ZP I konkretisiert. Nach Art. 36 ZP I muss jede neue Waffe anhand dieser Kriterien überprüft werden. Für ein generelles Verbot durch das HVR müsste der Einsatz von UCAVs nach Art. 36 ZP 1 per se gegen eine anwendbare Regel des Humanitären Völkerrechts verstoßen.

Dies ist zwar nicht ausgeschlossen, doch ebenso wenig automatisch durch den Einsatz eines UCAV gegeben. Vielmehr hängt dies von der eingesetzten Waffe oder dem Verhalten des Operators $\mathrm{ab}$, der sie sowohl in Einklang als auch im Widerspruch zum HVR einsetzen kann. Dem Trägersystem gegenüber verhält sich das HVR dagegen neutral, wie Völkerrechtler Michael N. Schmitt darlegt. ${ }^{14}$ Auch VN-Sonderberichterstatter Philip Alston betont:

„[A] missile fired from a drone is not different from any other commonly used weapon [...]. The critical legal question is the same for each weapon: whether its specific use complies with IHL [=International Humanitarian Law]. "15

Selbstredend sind hierbei die bestehenden völkerrechtlichen Verbote zu berücksichtigen, sodass etwa eine Bestückung einer Drohne mit Chemiewaffen klar gegen das HVR verstoßen würde. Ein generelles Verbot der Drohne als Trägersystem kommt dagegen jedoch nicht in Betracht. ${ }^{16}$

Durch den Einsatz von UCAVs sind, so Befürworter, sogar wesentlich zielgenauere Eingriffe möglich. Durch die LiveÜbertragung der Bilder kann der Steuernde zeitnah die Lage einschätzen und auf geänderte Umstände, wie z.B. sich nähernde Zivilisten, adäquat reagieren, während dies etwa bei einer einmal gezündeten Langstreckenrakete nicht der Fall ist.

13 Anders in konkreten Verbotsabkommen, die auch zum Humanitären Völkerrecht gezählt werden, wie etwa dem Chemiewaffenübereinkommen von 1993 und dem Ottawa-Abkommen über das Verbot von Personenminen von 1997.

14 Schmitt, Michael N., Drone Attacks under the Jus ad Bellum and Jus in Bello: Clearing the 'Fog of Law', in: Yearbook of International Humanitarian Law 2010, Chapter 9, S. 311, 322f.

15 Alston, Philip, Report of the UN Special Rapporteur on extrajudicial, summary or arbitrary executions, UN Doc A/HRC/14/24/Add. 6 (28.05.10), para 79: http:// www2.ohchr.org/english/bodies/hrcouncil/docs/14session/A.HRC.14.24. Add6.pdf (06.02.14).

16 Marauhn, Thilo, Der Einsatz unbemannter bewaffneter Drohnen im Lichte des geltenden Völkerrechts, in: Deutsche Stiftung Friedensforschung Arbeitspapiere DSF Nr. 9: Unbemannte bewaffnete Systeme: Verändert der rüstungstechnologische Wandel den Umgang mit Konflikten? Eine friedenspolitische Perspektive - Beiträge zum Parlamentarischen Abend der DSF am 25.09.12 in Berlin, S. 31.
Durch die räumliche Distanz erfolgt auch ein weitaus nüchternsachlicheres Bild bei dem bedienenden Operator. Emotionale Stressreaktionen (Wut, Rache etc.) oder Vergeltungsgedanken (Plünderungen, Vergewaltigungen etc.) könnten so reduziert und der oftmals unübersichtliche Kriegsschauplatz („Kriegsnebel") besser erfasst werden. ${ }^{17}$

Ein generelles Verbot des Einsatzes von UCAVs durch das HVR besteht daher nicht.

\subsection{Rüstungskontrolle von UCAVs - ein erster Schritt?}

Zur Begrenzung und Deckelung der Herstellung und Verbreitung von Kampfdrohnen kommen internationale Rüstungskontrollabkommen in Betracht.

Einer ausschweifenden Verbreitung soll präventiv zuvorgekommen werden, um zu verhindern, dass nichtstaatliche Akteure wie terroristische Gruppierungen in den Besitz dieser sensiblen Technik gelangen. ${ }^{18}$ Gleichzeitig warnen kritische Stimmen bei der ungehinderten Weiterentwicklung von UCAVs vor einem, nach ihrer Ansicht folgerichtigen Schritt hin zu mehr und letztlich absoluter Autonomie (off-the-loop). ${ }^{19}$ Begründet wird dies damit, dass die menschliche Reaktionszeit zum militärischen Nachteil erwachsen kann. Dem könne man letztlich nur mit automatisch reagierenden Systemen begegnen, um dem Gegner die entscheidende Sekunde voraus zu sein. ${ }^{20}$ Zugleich senke die große, räumliche Distanz zwischen steuerndem Mensch und angreifender Maschine die Hemmschwelle zum Einsatz. ${ }^{21}$

Andererseits bestehen aber bereits seit Jahrzehnten solche räumliche Distanzen durch immer größere Raketenreichweiten. Auch bleibt, so Marauhn, durch die räumliche Distanz und Sicherheit des Operators, ein größeres Zeitfenster zur Verarbeitung der Informationen und damit auch zur Einordnung und Bestimmung des Zielobjekts.

Zudem belegen diverse Studien bereits die psychischen Auswirkungen für solche „joy stick fighter", bei denen ähnliche posttraumatische Stress-Symptome auftreten, wie bei Soldaten, die tatsächlich auf dem fremden Territorium stationiert sind, ${ }^{22}$

17 Vgl. Sparrow, Robert, Predators or Plowshares? Arms Control of Robotic Weapons, Institute of Electrical and Electronics Engineers (IEEE) Technology and Society Magazine 1/2009, S. 25, 26: http://www.deepdyve.com/lp/instituteof-electrical-and-electronics-engineers/predators-or-plowshares-arms-controlof-robotic-weapons-pfFHemM5Cn (12.02.14).

18 Vgl. Taylor, 10.11.13.

19 Schörnig und Weidlich, 2013.

20 Vgl. Sauer, Frank und Schörnig, Niklas, Killer drones: The ,silver bullet' of democratic warfare?, Security Dialogue 43/2012, S. 363, 374: http://sdi.sagepub. com/content/43/4/363.full.pdf+html (10.02.14).

21 Vgl. Vogel, Ryan J., Drone Warfare and the Law of Armed Conflict, Denve Journal of International Law and Policy 39/2011, S. 101, 133: http://djilp. org/wp-content/uploads/2011/08/3-Vogel.pdf (10.02.14).

22 Vgl. Chow, Denise, Drone Wars: Pilots Reveal Debilitating Stress Beyond Virtual Battlefield, Live Science, 05.11.13: http://www.livescience.com/40959military-drone-war-psychology.html (10.02 14); siehe auch: Thompson, Marc, Drone Pilots: No Worse Off Than Those Who Actually Fly, Time, 02.04.13: http://nation.time.com/2013/04/02/drone-pilots-no-worse-off-than-thosewho-actually-fly/ (10.02.14); Studie: Otto, Jean L und Webber, Bryant J, Mental Health Diagnoses and Counseling Among Pilots of Remotely Piloted Aircraft in the United States Air Force, Medical Surveillance Monthly Report 3/2013, S. 3ff: http://timemilitary.files.wordpress.com/2013/04/pages-from-pages-frommsmr_mar_2013_external_causes_of_tbi.pdf (10.02.14). 
was das Sinken einer Hemmschwelle, jedenfalls für den Operator selbst, in Zweifel zieht.

Dass ein hohes Potenzial zur schnellen Verbreitung, auch an außerstaatliche Akteure, besteht, ist dagegen ein durchaus ernst zu nehmendes Problem. Vor allem durch den - im Vergleich zu bemannten Kampfflugzeugen - kostengünstigen und die eigenen Truppen schonenden Einsatz, ist mit einem vermehrten Rückgriff zu rechnen. Auch gezielte terroristische Anschläge ließen sich so auf vergleichsweise günstige Weise und ohne eigene Opfer und damit geringerem Aufwand durchführen.

Im Folgenden wird daher ein Blick auf bereits bestehende Rüstungskontrollabkommen geworfen und dargestellt, inwieweit auch UCAVs von diesen bereits erfasst werden.

\subsubsection{KSE-Vertrag}

Eines der wichtigsten Rüstungskontrollabkommen der jüngeren Geschichte ist der Vertrag über Konventionelle Streitkräfte in Europa (KSE-Vertrag), der 1990 unterzeichnet wurde und zwei Jahre später in Kraft trat.

Darin verpflichteten sich die dreißig Mitgliedstaaten der NATO und des damaligen Warschauer Pakts auf eine mengenmäßige Begrenzung konventioneller Waffen in fünf Kategorien. Das 1999 verhandelte Istanbuler Übereinkommen über die Anpassung des KSE-Vertrags wurde jedoch lediglich von Russland und drei weiteren Staaten ratifiziert. Die NATO-Staaten verweigerten die Ratifizierung, weil Russland auf der Istanbuler Konferenz gemachte Zusagen nicht einhielt. Dieser Konflikt gipfelte 2007 in einer Aussetzung des KSE-Vertrags durch Russland.

Ob UCAVs unter das Rüstungskontrollabkommen fallen, ist umstritten. Während etwa die letzte deutsche Bundesregierung $^{23}$ ebenso wie Verteidigungsexperte Klaus Wittmann ${ }^{24}$ eine solche Interpretation nicht erkennen, widersprechen dem Abrüstungsexperten wie Jürgen Altmann ${ }^{25}$ und der SWP-Forscher Wolfgang Richter ${ }^{26}$ überzeugend.

So wird im KSE-Vertrag ein „Kampfflugzeug“ in Art. II Abs. 1 (K) als

„[...] Starrflügel- oder Schwenkflügelflugzeug, das für die Bekämpfung von Zielen durch den Einsatz von gelenkten Flugkörpern, ungelenkten Raketen, Bomben, Bordmaschinengewehren, Bordkanonen oder anderen Zerstörungswaffen bewaffnet und

23 Vgl. Antwort der Bundesregierung auf die Kleine Anfrage der Abgeordneten Agnes Brugger, Volker Beck (Köln) Marieluise Beck (Bremen), weiterer Abgeordneter und der Fraktion BÜNDNIS 90/DIE GRÜNEN, Abrüstungsund Rüstungskontrollpolitik, Deutscher Bundestag, Drucksache 17/11956 (20.12.12), Frage 14: http://dipbt.bundestag.de/dip21/btd/17/119/1711956. pdf (11.02.14).

Später nicht mehr ganz so deutlich ablehnend: Antwort der Bundesregierung auf die Kleine Anfrage der Abgeordneten Andrej Hunko, Jan van Aken, Herbert Behrens, weiterer Abgeordneter und der Fraktion DIE LINKE, Militärische Drohnen-Strategie der Bundesregierung: Kampfdrohnen, Deutscher Bundestag, Drucksache 17/14053 (20.06.13), Frage 20 b: http://dipbt.bundestag.de/ dip21/btd/17/140/1714053.pdf (11.02.14)

24 Vgl. Wittmann, Klaus, Mehr Differenzierung, bitte!, The European (12.07.13): http://www.theeuropean.de/klaus-wittmann/7171-noetige-entscheidungenzur-drohnen-debatte (11.02.14)

25 Vgl. Richter, Torben, Interview: TU-Physiker korrigiert Bundesregierung, Interview mit Jürgen Altmann, Pflichtlektüre (11.02.13): http://www.pflichtlektuere.com/11/02/2013/interview-dr-altmann/ (11.02.14).

26 Vgl. Richter, 29.05.13, S. 2 f. ausgerüstet ist, sowie jedes Modell oder jede Version eines solchen Flugzeugs, das andere militärische Aufgaben wie z.B. Aufklärung oder elektronische Kampfführung wahrnimmt. [...]“

definiert. Die Erwähnung einer Besatzung erfolgt dabei im Gegensatz zu anderen Stellen im Abkommen nicht. ${ }^{27}$ Daraus lässt sich im Wege eines argumentum e contrario ableiten, dass die fehlende Erwähnung einer Besatzung bewusst erfolgte, zumal in den 1990er Jahren solche Drohnen durchaus bereits in militärischen Kreisen bekannt waren. Beim KSE-Vertrag zählte eine möglichst umfassende Formulierung, um Schlupflöcher auf beiden Seiten zu verhindern. Daher fallen auch unbemannte Flugzeuge unter die besagte Definition.

Warum aber sind solche Kampfdrohnen bis dato nicht auch in der Praxis in Einklang mit dem KSE-Statut kontrolliert und registriert worden? Hierfür führt Richter ${ }^{28}$ gleich mehrere Gründe an: Einerseits fand bisher keine Stationierung auf dem vertragsrelevanten Gebiet der USA oder der europäischen Mitgliedstaaten statt, sondern lediglich im afrikanischen oder asiatischen Ausland. Zum anderen ist durch die russische Aussetzung das Abkommen weder angepasst worden, noch besteht der politische Wille, sich selbst zu binden, ohne dass Russland vergleichbare Maßnahmen ergreift.

\subsubsection{MTCR}

Das Missile Technology Control Regime (MTCR) wurde 1987 von den G-7-Staaten gegründet. Der anfängliche Zweck der Durchsetzung eines Waffenembargos gegenüber den Staaten aus der sowjetischen Einflusssphäre wich mit Fall des „Eisernen Vorhangs" einem internationalen Exportkontrollsystem, dem inzwischen 34 Staaten, wie Russland, Brasilien oder Südafrika angehören.

Dabei steht die Eindämmung des Handels mit Langstreckenraketen, Trägersystemen und weiteren Dual-use-Komponenten im Fokus. Dieser Handel soll nicht nur registriert, sondern möglichst unterbunden werden, wobei die von den Mitgliedstaaten ausgearbeiteten Richtlinien nicht verpflichtend sind und von jedem Mitglied in das eigene Rechtssystem implementiert werden müssen.

Auch UAVs fallen unter das MTCR und werden in sogenannte Kategorie 1 (most sensitive) und Kategorie 2 (less sensitive) eingeteilt. Lediglich solche mit einer Reichweite von mehr als $300 \mathrm{~km}$ fallen unter Kategorie 2, während für Kategorie 1 neben der Mindestreichweite eine Nutzlast von nicht unter $500 \mathrm{~kg}$ bestehen muss. Hierdurch fällt bereits eine Vielzahl heute eingesetzter UAVs aus dem Raster.

\subsubsection{Wassenaar-Abkommen}

Das Wassenaar-Abkommen (WA) von 1996, das bereits von 41 Staaten unterzeichnet wurde, soll zur Exportkontrolle von

27 So etwa bei "gepanzerten Mannschaftstransportwagen“ (Art. 2 Abs. 1 (D) Unterabsatz 1 KSE-Vertrag) und „Schützenpanzern“ (Art. 2 Abs. 1 (D) Unterabsatz 2 KSE-Vertrag).

28 Vgl. Richter, 29.05.13, S. 2 f. 
konventionellen Waffen und Dual-use-Gütern und -Technologien beitragen. Die Staaten entscheiden zwar eigenständig über die Exportanfragen, doch werden Bewilligung oder Ablehnung den anderen Staaten mitgeteilt. Dadurch soll verhindert werden, dass von einem Mitgliedsland abgelehnte Anfragen von anderen positiv beschieden werden.

Auch UAVs fallen bereits unter den Anwendungsbereich des WAs. So tauchen sie sowohl in der „List of Dual-use Goods and Technologies“29 als auch in der „Munitions List“30 auf. Damit ist die Definition des WA wesentlich weiter als etwa das MTCR und bezieht so auch solche UCVs mit ein, die einen kleineren Radius haben. Gleichzeitig ist das WA jedoch kein Instrument zur direkten Abrüstung, sondern hat lediglich Transparenz- und Koordinierungsfunktion, um ggf. eine Nichtverbreitung an bestimmte Staaten durchzusetzen.

Dennoch ist es wichtig, dass hier ein Abkommen vorliegt, das UAVs ausdrücklich erwähnt und deren problematischen Dual-use-Charakter hervorhebt. Zu einem ähnlichen Schluss kommt auch ein Bericht des Bundestagsausschusses für Bildung, Forschung und Technikfolgenabschätzung aus dem Jahr 2011. Dieser wertete das WA als guten „Ansatzpunkt [...] für eine Begrenzung des Exports [...]" von UAVs. ${ }^{31}$

\subsubsection{VN-Waffenregister und Arms Trade Treaty}

Das VN-Waffenregister (Convention on Prohibitions or Restrictions on the Use of Certain Conventional Weapons Which May Be Deemed to Be Excessively Injurious or to Have Indiscriminate Effects, CCW) wurde im Jahr 1991 durch eine Resolution der Generalversammlung der VN beschlossen. Hiernach sollen die Mitgliedstaaten der VN jährlich Informationen über Exporte und Importe konventioneller Großwaffensysteme ${ }^{32}$ liefern

29 Item 9. A. 12. "Unmanned Aerial Vehicles" (UAVs"), unmanned "airships", related systems, equipment and components, as follows:

a. "UAVs" or unmanned "airships", having any of the following:

1. An autonomous flight control and navigation capability (e.g., an autopilot with an Inertial Navigation System); or

2. Capability of controlled flight out of the direct visual range involving a human operator (e.g., televisual remote control);

b. Related systems, equipment and components, as follows:

1. Equipment specially designed for remotely controlling the "UAVs" or unmanned "airships", specified by 9.A.12.a.;

2. Systems for navigation, attitude, guidance or control, other than those specified by Category 7, specially designed to be integrated into "UAVs" or unmanned "airships", specified by 9.A.12.a.;

3. Equipment or components, specially designed to convert a manned "aircraft" or a manned "airship" to a "UAV" or unmanned "airship", specified by 9. A.12.a.;

Item 9. A. 12. b. 4. Air breathing reciprocating or rotary internal combustion type engines, specially designed or modified to propel "UAVs" or unmanned "airships", at altitudes above 50,000 feet (15,240 meters).

30 ML10. "Aircraft", "lighter-than-air vehicles", "Unmanned Aerial Vehicles" ("UAVs"), aeroengines and "aircraft" equipment, related equipment, and components, as follows, specially designed or modified for military use: c. Unmanned aircraft and related equipment, as follows, and specially designed components there for:

1. "UAVs", Remotely Piloted Air Vehicles (RPVs), autonomous programmable vehicles and unmanned "lighter-than-air vehicles";

2. Launchers, recovery equipment and ground support equipment;

3. Equipment designed for command or control.

31 Petermann, Thomas und Grünwald, Reinhard (Büro für TechnikfolgenAbschätzung beim Deutschen Bundestag), Stand und Perspektiven der militärischen Nutzung unbemannter Systeme - Endbericht zum TA-Projekt, Mai 2011: http://www.tab-beim-bundestag.de/de/pdf/publikationen/berichte/ TAB-Arbeitsbericht-ab144.pdf (10.02.14).

32 Hierzu gehören die sieben Kategorien Kampfpanzer, gepanzerte Kampffahrzeuge, großkalibrige Artilleriesysteme, Kampfflugzeuge, Kampfhubschrauber, Kriegsschiffe und Raketen mit deren Start- und Abschusssystemen. und so die zwischenstaatliche Vertrauensbildung durch Transparenz fördern. Auch Waffenbestände, Eigenproduktionen, Kleinwaffentransfers oder Leitlinien zur Abrüstung können auf freiwilliger Basis übermittelt werden.

Zum letzten Stichtag lieferten insgesamt 61 Staaten Informationen, darunter China, die USA und Russland. Aufgrund der erwähnten Freiwilligkeit sind diese jedoch unterschiedlich vollständig. ${ }^{33}$ Zudem ist die Zahl der Staaten, die sich tatsächlich beteiligen, in den letzten Jahren rückläufig. ${ }^{34}$

Der im April 2013 geschlossene Arms Trade Treaty (ATT), der nach 50 Ratifizierungen in Kraft treten wird, ${ }^{35}$ ist der erste umfassende völkerrechtliche Vertrag zur verbindlichen Regulierung des globalen Waffenhandels. Waffenexporte an Staaten oder Gruppierungen, denen Menschenrechtsverletzungen und Kriegsverbrechen vorgeworfen werden, sollen so beispielsweise unterbunden werden. Dabei bezieht sich der ATT auf die Waffenkategorien des VN-Waffenregisters, die entsprechend gelten. ${ }^{36}$

Eine Einbeziehung von UCAVs in das Register würde somit ebenso für den ATT gelten. Eine Gruppe von UN-Experten kam hierbei im Juli letzten Jahres zu dem Ergebnis, dass UCAVs bereits unter die Kategorien „Kampfflugzeuge“ und „Kampfhubschrauber" fallen, da - ähnlich wie beim Wassenaar-Abkommen - von einer Besatzung keine Rede ist. ${ }^{37}$ Dennoch empfahl die 15-köpfige internationale Gruppe eine Erweiterung der bestehenden Definition um Unklarheiten zu vermeiden. Zwar versicherten viele Staaten eine weite Auslegung des Vertragstextes ${ }^{38}$, doch bleiben bei einer fehlenden Ausformulierung Zweifel, ob alle Mitgliedstaaten einer solchen Interpretation folgen.

\section{Fazit}

Dass der Einsatz von Drohnen eine wachsende Rolle bei der Kriegführung im 21. Jahrhundert spielen wird, steht außer Frage. Hierfür sprechen vor allem innenpolitische Gründe, wie die niedrigen Kosten im Vergleich zu Kampfjets und der Schutz der eigenen Soldaten. Widerständen innerhalb der eigenen Bevölkerung gegen militärische Einsätze könnte durch Minimierung von Kosten und eigenen Opfern wesentlich entgegengewirkt werden.

Auch ließe sich der Trend hin zu asymmetrischen Konflikten und weg von klassischen Zwei-Staaten-Kriegen mit solchen Kriegsmitteln beantworten. Ein reiner Einsatz von UCAVs scheint dagegen weniger realistisch. Aufgrund ihrer vergleichsweise großen Anfälligkeit etwa gegen Luft-Luft-Raketen und

33 Deutschland lieferte etwa Daten über Waffenexporte, -importe, Bestände, Eigenproduktionen, Kleinwaffen-Transfers und seine Abrüstungspolitik, während China dagegen lediglich seine Export-Daten (und das Fehlen von Importen) übermittelte.

34 Vgl. Morley, Jefferson, UN Experts Call for Drone Reporting, in: Arms Control Today 43/2013: https://www.armscontrol.org/act/\%252F2013 09/UNExperts-Call-for-Drone-Reporting (14.02.14).

35 Derzeit haben elf Staaten den ATT ratifiziert (18.02.14).

36 Art. 2 (1) des ATT.

37 Bindseil, Wolfgang Jakob et al., Report on the continuing operation of the United States Register of Conventional Arms and ist further development, UN General Assembly, UN Doc A/68/140 (15.07.13), paras 45, 46 und 69: http://www. un.org/ga/search/view_doc.asp?symbol=A/68/140 (14.02.14).

38 Vgl. Bolton, Matthew und Zwijnenburg, Wim, Futureproofing: Making Sure the Arms Trade Treaty Controls Drones and New Robotic Weapons, in: Policy Wonk's Corner (Control Arms Blog), 07.11.13: http://controlarmsblog. wordpress.com/2013/11/07/futureproofing-making-sure-the-arms-tradetreaty-controls-drones-and-new-robotic-weapons-2/ (14.02.14). 
niedriger Geschwindigkeit ist eine reine Abstützung auf UCAVs bei der konventionellen Kriegführung in naher Zukunft jedenfalls nicht zu erwarten.

Was eine Ächtung vollautonomer UCAVs angeht, sind die Beratungen des CCW im Mai 2014 ein erster Schritt auf einem noch langen Weg. Die fehlende Einigung über das Protokoll VI zu Streumunition zeigt, wie die Diskussionen zwar in einer Sackgasse enden können. Doch werden so auch pragmatische Lösungen möglich, wie das separate Übereinkommen über Streumunition gewillter Staaten zeigt. ${ }^{39}$ Eine besondere Schwierigkeit liegt bei den vollautonomen UCAVs jedoch - im Gegensatz zu vorherigen Protokollen - in der Definition des zu ächtenden Systems.

So war bei vorangegangenen Verboten eine konkrete Bestimmung möglich, zumal sich diese immer auf eine bestimmte Hardware und deren Wirkung bezog. Was biologische und chemische Waffen oder Antipersonen-Minen sind, kann klarer abgegrenzt werden. Bei vollautonomen UCAVs käme es dagegen neben der Bewaffnung im Wesentlichen auf die Programmierung an. Doch wo beginnt die volle Autonomie?

Ist eine selbständig fliegende und schießende Drohne, die aber ausgeschaltet oder vom Operator zerstört werden kann, autonom?

Eine Abgrenzung bei der Programmierung zu ziehen, die ausreichende Klarheit bietet, birgt eine besondere Herausforderung. Dass bis dato keine vollautonomen UCAVs existieren, macht eine Definition zwar nicht unmöglich, aber doch ungleich komplizierter. Dennoch muss die internationale Politik hier „am Ball bleiben“ und technologische Fortschritte genauestens analysieren. Vor allem sollte daher im Mai 2014 darauf hingewirkt werden, eine Definition zu erarbeiten und den Stand der Entwicklung etwa durch regelmäßige Berichte einer Expertenkommission zu verfolgen, um adäquat reagieren $\mathrm{zu}$ können. Nationale Moratorien, wie in den USA, können einen Beitrag leisten, doch führt an einer gemeinsamen Antwort der internationalen Gemeinschaft kein Weg vorbei.

Eine zeitnahe Umsetzung ist hingegen bei Rüstungskontrollabkommen in Bezug auf UCAVs wesentlich eher realisierbar und auch geboten. Die rasante technische Entwicklung macht Kontrollinstrumente in Form von internationalen Abkommen dringend erforderlich. So ist die Sorge vor einer unkontrollierten Verbreitung in kürzester Zeit durchaus berechtigt, wie die Dynamik des Drohnenmarktes der letzten Jahre zeigt. Damit besteht auch eine erhöhte Gefahr, dass diese Technik in „falsche Hände“ gerät. Der technologische Vorsprung der USA und Israels wird sich dauerhaft nicht halten können, was den betreffenden Staaten bewusst werden muss. Um neuen Rüstungswettläufen vorzubeugen, sollte daher verstärkt auf Transparenz gesetzt werden, wie auch UN-Sonderberichterstatter Christof Heyns fordert. ${ }^{40}$ Eine ausreichende Basis

39 Convention on Cluster Munitions von 2010: http://www.clusterconvention. org/.

40 "The first step towards securing human rights in this context is transparency about the use of drones. A lack of appropriate transparency and accountability concerning the deployment of drones undermines the rule of law and may threaten international security." Heyns, Christof, Report of the Special Rapporteur on extrajudicial, summary or arbitrary executions, UN General Assembly, UN Doc A/68/382 (13.09.13), para 96 f.: http://justsecurity.org/ wp-content/uploads/2013/10/UN-Special-Rapporteur-Extrajudicial-ChristofHeyns-Report-Drones.pdf (11.02.14). liegt bereits mit bestehenden Abkommen vor, sodass der Abschluss neuer Rüstungskontrollabkommen für UCAVs kaum erforderlich ist.

Eine neue Initiative zur Anpassung des KSE-Vertrags könnte auch UCAVs umfassen. Bei deren Entwicklung hält Russland bisher nicht mit „dem Westen“ Schritt, sodass ein Anreiz zur Wiederaufnahme der Verhandlungen geschaffen werden könnte. Dass eine solche Initiative Früchte trägt, ist beim derzeitigen politischen Klima noch nicht absehbar. Auch stehen für Russland militärische Projekte, wie der von den USA geplante Raketenabwehrschirm in Polen und Tschechien, weitaus höher auf der Bedrohungsagenda. Dennoch könnte eine solche Initiative einen Beitrag zur politischen Entspannung leisten.

Für das Wassenaar-Abkommen sollte auf eine gemeinsame, verbindliche Erklärung aller Mitgliedstaaten hingearbeitet werden, um bestehende Unklarheiten bei der Einbeziehung von Drohnen zu beseitigen. Beim MTCR-Abkommen könnte zusätzlich eine Verringerung der erforderlichen Spezifikationen fokussiert werden, sodass auch kleinere UCAVs erfasst werden. Zugleich müssen die Bemühungen verstärkt werden, auch Staaten, wie Israel, China oder den Iran, die bereits jetzt im Besitz von UCAVs bzw. des Besitzes verdächtig, aber nicht Mitglieder des Wassenaar- oder MTCR-Abkommens sind, zur Ratifizierung zu bewegen.

Ähnlich steht es um das VN-Waffenregister und den ATT. Die Entwürfe einer verbindlichen Definition, die der VN-Generalversammlung durch die Kommission unterbreitet wurden, sollten zur eindeutigen Klärung des Status von UCAVs in die Tat umgesetzt werden. Hierdurch wäre mit dem VN-Waffenregister, an dem sich die wichtigsten Waffenexporteure beteiligen, ein großer Schritt zur weltweiten Transparenz getan. Auch könnte ein solcher Impuls für weltweite Transparenz der negativen Beteiligungstendenz der letzten Jahre entgegenwirken. Zugleich würde synchron eine Implementierung in den ATT erfolgen. Dadurch wäre ein weiterer wichtiger Schritt getan, den ATT für die Zukunft als zentrales Vertragswerk zur Regelung des transnationalen Waffenverkehrs zu etablieren. 